\title{
Acoustic Emission Signal Analysis for the Integrity Evaluation
}

\author{
Tomaž Kek1, ${ }^{*}$ - Dragan Kusić ${ }^{2}$ - Rajko Svečko ${ }^{3}$ - Aleš Hančič2 - Janez Grum ${ }^{1}$ \\ 1 University of Ljubljana, Faculty of Mechanical Engineering, Slovenia \\ 2 TECOS Slovenian Tool and Die Development Centre, Slovenia \\ 3 University of Maribor, Faculty of Electrical Engineering and Computer Science, Slovenia
}

This paper presents measurements of acoustic emission (AE) signals during injection molding with resonant PZT sensors that were applied to the mold via waveguides. A polypropylene material was employed for injection molding of ISO specimens. Acoustic signals were measured during production cycles on a new mold and damaged one with cracks induced by laser surface heat treatment. The mold inserts integrity description by acquired AE signal together with the fractal algorithm using box counting method is presented. Implementation of AE signal analysis based on an idea of the box-counting method in a way to divide the measured AE signals to AE signal boxes is used. To improve the capability of clustering AE data during injection process cycle, AE burst descriptors are defined. To lower computational complexity and increase performance, the feature selection method was implemented. Neural network pattern recognition of AE signals feature subsets was used for evaluation of process steps and damage detection.

Keywords: injection molding, acoustic emission, cracks, box counting method, pattern recognition

Highlights

- A method is presented able to evaluate integrity of injection molding tool based on signal acquisition with resonant sensors.

- Different aspects of signal analysis of acquired acoustic signals of a steel insert are used for integrity evaluation.

- Implementation of signal analysis based on idea of box counting method in a way to divide the measured acoustic signals to signal boxes is used.

- Implemented signal burst analysis with burst descriptors improves the capability of clustering the measured data during injection cycle. To lower computational complexity and increase performance of the evaluation, the feature selection method was implemented.

\section{O INTRODUCTION}

Injection molding process is very popular manufacturing process for production of plastic products. It offers production of complex shaped products with high tolerance requirements which is especially difficult with combination of different polymer material. Typical production cycle usually incorporate mold closing followed by injection of molten material into a cavity. After filling the cavity, the packing pressure is applied to compensate for the material shrinkage. Product has to be sufficiently cooled for mold opening and ejection of the part.

The major acoustic signal sources detected by nondestructive testing (NDT) are crack growth and plastic deformation of the steel [1]. Acoustic inspection offers determination of location and propagation of a crack during loading as already reported by many researchers that are working in various fields. Acquisition of acoustic emission (AE) signals during different production processes in different setups has become widely used because of high sensitivity and usefulness for online surveillance [2] to [4]. Cao et al. [5] investigated acoustic signals during four-point bending fatigue crack propagation in steel. In the first stage of fatigue damage process a dominant acoustic source was micro-crack initiation.
During intermediate stage of crack growth, stacking and slipping of dislocations ahead of the crack tip are major acoustic sources. During final stage the predominant acoustic sources are shearing of ligaments and connectivity between dimples. They have set neural network model with input time domain parameters based on burst energy, peak amplitude, duration, and counts. Mukhopadhyay, et al. [6] analyzed these signals during fracture toughness tests with a compact tension (CT) specimens made of steel. During fracture toughness tests the signals have been attributed to the plastic zone formation at the crack tip and initiation and/or extension of crack. Drummond et al. [7] analyzed enhancement of proof and fatigue testing procedures for wire ropes with incorporation of measurement of AE signals. The results of their research showed that the most effective AE signal discriminators are peak amplitude and (cumulative) burst energy for differentiation between signals from wire breaks and other sources. For determination of the rope's condition there is no need for continuous monitoring and also measurement of $\mathrm{AE}$ is indicative of the level of damage of the rope. Kim et al. [8] measured fatigue crack growth with standard CT specimens using a hydraulic loading machine. The result of their research was the development of a neural network based model used for the prediction 
of stress intensity factor based on five parameters of acoustic emission signals (peak amplitude, energy, ring down count, rise time and event duration). Their results showed gradual increase of AE energy with the number of fatigue cycles. Change in the acoustic emission energy with the number of fatigue cycles was an effective parameter for estimation of the activity of the crack propagation and the stress intensity factor.

Acoustic emission monitoring offers a big potential for detailed in situ failure analysis of different materials. Measurement of AE signals provides information on sub-macroscopic failure phenomena and also on the overall damage accumulation [9] to [11]. Measured AE signals contain useful information on the damage mechanism in a broad spectrum of applications [12]. Measurement of AE signals during production processes or during loading of different structures usually generates a high number of detected signals. These signals can be associated to a patterns (vectors) composed of multiple relevant descriptors [13] to [16] with the intention to discriminate different damage mechanisms described with clusters. The patterns (vectors) can be classified into clusters according to their similarity by the use of multivariable data analyses based on pattern recognition algorithms [17]. Piezoelectric (PZT) sensors are usually used for inspection. They can measure ultrasound with a high sensitivity $(\sim 1 \mathrm{~V} / \mathrm{nm})$ as a displacement sensors and a few $\mathrm{V} /(\mathrm{mm} / \mathrm{s})$ as a velocity sensors. Since they are sensitive only on dynamic events they automatically compensate low-frequency motion of measuring objects that can be caused by environmental vibrations.

Low-frequency cutoff of PZT sensors is a consequence of the leakage of the accumulated charge and acts as a high-pass filter. The low-frequency cutoff is determined through a time constant given by the capacitance and resistance of the device [18]. Additionally to above mentioned advantages, PZT sensors are also insensitive to electromagnetic fields and radiation, enabling measurements under harsh conditions.

\section{METHODS}

Correlation of crack propagation in steel and basic acoustic burst signal parameters is well described in the literature but on the other hand the detection of tool damage in the literature didn't gained sufficient coverage in the past.

In this paper different aspects of mold (tool insert) integrity based on acquired AE signals are presented. In the section 3.1 description of tool insert integrity by time domain acoustic signal descriptors together with fractal algorithm using box counting method is presented. Section 3.2 describes implementation of acoustic signal analysis based on idea of box counting method in a way to divide the measured signals to "signal boxes". Prediction of the tool insert damage is based on signal frequency characteristics and signal amplitude probability distribution into the decision criteria. The signal box can be an aggregate of information that is described by individual bursts. Section 3.3 thou describes the analysis of acquired acoustic signals in a form of bursts. The burst descriptors are stacked to form a feature (measurement) vector Z. After signal processing neural network pattern recognition of bursts during the full time of the injection molding cycle is presented.

\section{EXPERIMENTAL}

For injection molding one cavity tool for production of square test specimens with dimensions of $60 \mathrm{~mm}$ $\times 60 \mathrm{~mm} \times 2 \mathrm{~mm}$ was used. These specimens can be used for variety of tests according to ISO 294-3 standard [19]. For injection molding a polypropylene (PP) material isofil H40 C2 F NAT manufactured by Sirmax was used. Fig. 1 shows the used KraussMaffei KM 80 CX-SP 380 injection molding machine on which a mold with a steel mold insert made of OCR12VN steel is placed. Experiments were conducted using a new tool and a damaged tool, respectively. For generation of surface cracks on a damaged mold local laser surface hardening of the tool steel was used. Experiments were conducted on injection molding machine KraussMaffei KM 80 CX-SP 380 with application of a broad spectrum of

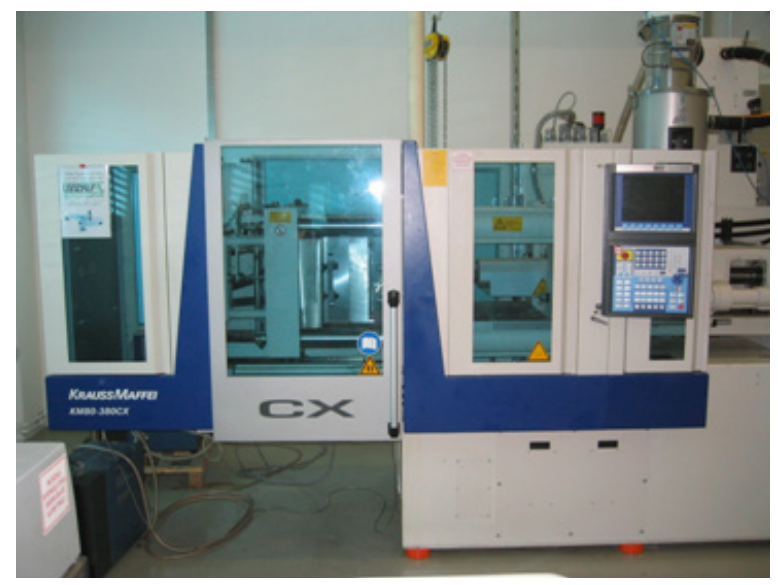

Fig. 1. The used injection molding machine KraussMaffei KM 80 CX-SP for experimental trials 
injection molding process parameters. For acoustic measurements Vallen AMSY-5 system was used with two resonant piezoelectric AE sensors VS150-M with a measuring range between $100 \mathrm{kHz}$ and $450 \mathrm{kHz}$ and resonance at $150 \mathrm{kHz}$. Sensors are connected via AEP4 preamplifiers with a gain of $40 \mathrm{~dB}$. These sensors cover frequencies spectrum that is characteristic for the signals generated during fatigue and plastic deformation [20]. For measurement an $18 \mathrm{-kHz}$ high pass filter was used. The amplitude threshold was set at $40 \mathrm{~dB}$ and sampling frequency to $5 \mathrm{MHz}$. To protect sensors against high temperatures, fumes exhaled out of the mold and to prevent mechanical damage during operation by the operator, additional waveguides were used.

\section{RESULTS AND DISCUSION}

\subsection{Burst AE Signals during Injection Molding Production Cycle}

Acoustic emission testing can detect dynamic processes associated with the degradation of structural integrity. During injection molding process a rapid increase of pressure during the filling and holding stage in mold occurs. This can stimulate defects in the mold that can result in release of AE signals in the form of burst.
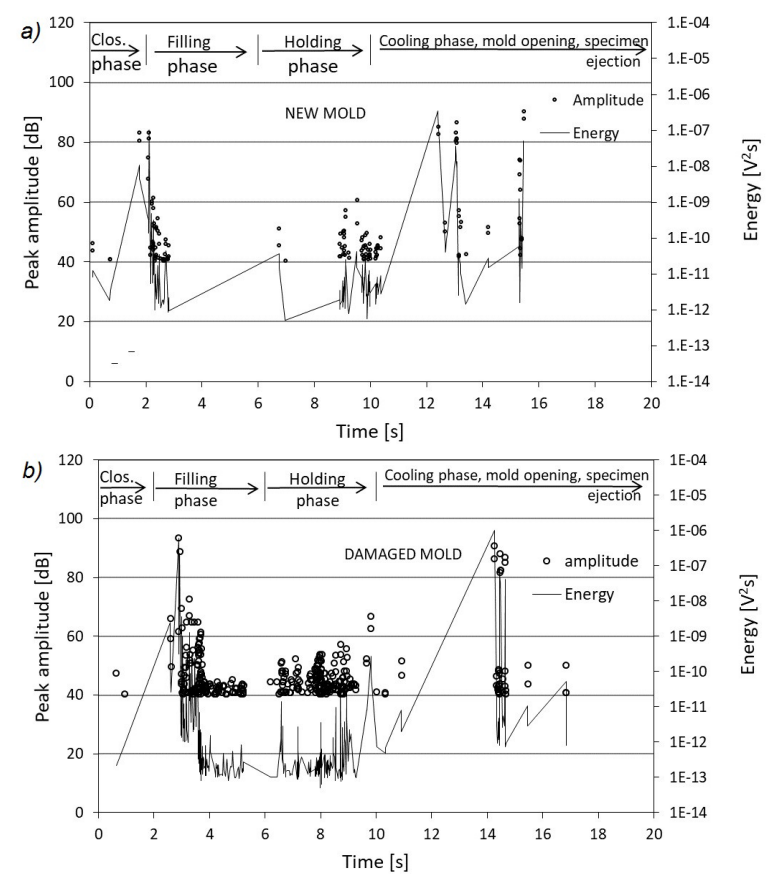

Fig. 2. Peak amplitudes and energy of AE bursts during the manufacturing of test specimens produced with a) the new and b) damaged mold
Basic AE parameters as peak amplitudes and energy of AE bursts measured during the production cycle of injection molding is shown on Fig. $2 \mathrm{a}$ and $\mathrm{b}$ for new mold and damaged mold. With a new intact mold absence of cracks offers us classifying detected acoustic signals as process orientated signals. Presence of damage in mold causes a considerable increase in the number of bursts especially during the filling and holding phase of the production cycle. Additional signals during these stages can consequently, be connected to active $\mathrm{AE}$ sources from mold defects. A rapid increase and high pressure in mold can stimulate plastic zone formation, slipping and stacking of dislocations in front of the crack tip and crack extension that can cause distinctive $\mathrm{AE}$ bursts [5], [6] and [21].

AE signal processing for evaluation of the defect in the injection mold can be achieved with fractal algorithm using box counting method. With this method acquired acoustic emission during injection molding cycle can be considered as time discrete signal. With setting time leg, also called ruler with different dimensions, the whole temporal window can be divided in an integer number of rulers (Fig. $3)$. Whenever the time leg $\mu$ contains a burst with the amplitude above the specified threshold, we can add 1 to the counter $G(\mu)$. Richardson's diagram incorporates interpolation line of $G(\mu)$ versus $\mu$ in a $\log$-log diagram. Absolute value of the $\tan (\varphi)$ can be called fractal dimension that can be used as damage parameter.

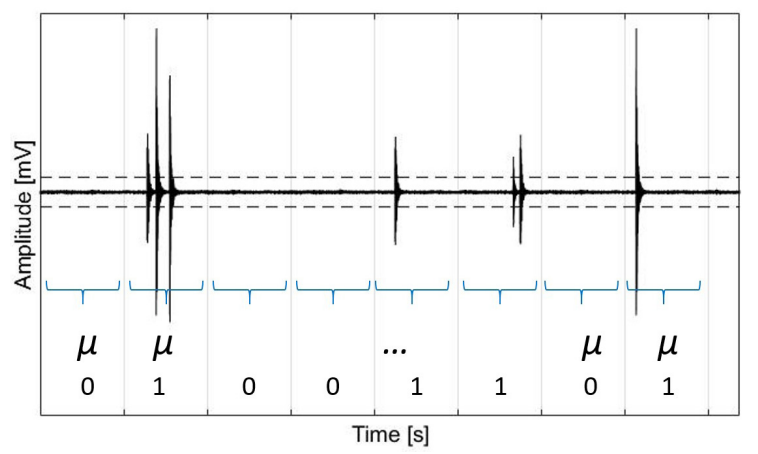

Fig. 3. Temporal window of acquired acoustic emission can be divided in an integer number of rulers

Fig. 4 shows that the presence of damage in the mold influences the slope of the interpolating line at a box counting method (BCM). Interpolated line designated as process is calculated according to the time discrete signal measured during the injection molding cycle with a new mold whilst interpolated line designated as process + damage describes 
injection molding cycle with a damaged mold. With a use of neural network pattern recognition captured $\mathrm{AE}$ burst can be classified into classes and signals connected with a damage, can be extracted. More about that will be described in the chapter 3.3. If we extract signals connected with damage in the mold we can calculate interpolated line designated as damage.

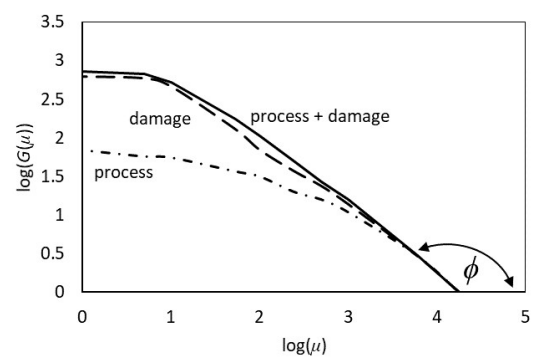

Fig. 4. Richardson's diagram for a new mold and damaged mold

\subsection{Analysis of Acquired Signals with Defined Time Length (AE Signal Boxes)}

The decision about the acceptability of a damaged mold with a small crack based basic AE parameters, burst rate and cumulative number of signal bursts is difficult. It is therefore reasonable to introduce additional features of detected signals during the production cycle in a form of acoustic signal frequency characteristics and amplitude probability distribution.

In our research we used the idea of box counting method in a way to divide the measured signals to "signal boxes" with defined time length (duration). Definition of signal boxes with defined time duration reduces computational complexity. For signal boxes we defined time length of $52.4 \mathrm{~ms}$ to cover also long bursts or burst cascades expected during cycle. Signal box represents signal vector $\mathbf{X}=\left(x_{1}, x_{2}, \ldots, x_{n}\right)$ where $n=262144$. We used $3.2 \mathrm{~ms}$ rearm time and 3.1998 ms duration discriminant time.

For signal boxes we calculated power spectrums using fast Fourier transform (FFT) with adjusted amplitude values below 1 unit and a frequency step of $19 \mathrm{~Hz}$. FFT is the most popular method for spectral analysis in digital signal processing [22] and [23]. Power spectral density is a measure of energy at various frequencies and is calculated based on FFT and the complex conjugate of FFT. We used Eq. (3) to calculate the $P_{L}$ parameter to characterize the lower part of the power spectrum $(a=90 \mathrm{kHz}, b=190 \mathrm{kHz})$. The higher part of power spectrum $(a=250 \mathrm{kHz}, b=$ $350 \mathrm{kHz})$ is characterized with parameter $P_{H}$, while $P_{S}$ covers all power spectrum frequencies $(a=50 \mathrm{kHz}$, $b=550 \mathrm{kHz}$ ). The parameters $P_{L}, P_{H}$ and $P_{S}$ give information about the energies at various frequencies inside of measured frequency spectrum.

$$
\begin{gathered}
g_{i}=\frac{y \overline{y i}}{m}, \\
m=\max (\mathbf{Y} \cdot \overline{\mathbf{Y}}), \\
P_{L}=\sum_{a=90}^{b=190} g_{i} .
\end{gathered}
$$

In above equations $\mathbf{Y}=\left(y_{1}, y_{2}, \ldots, y_{n}\right)$ is the vector of discrete Fourier transform using FFT.

Additional parameters describing the amplitude distribution of recorded signals like the kurtosis $K$ and skewness $S$ were introduced to improve the prediction of mold integrity. $K$ is a measure of the "peakedness" of the probability distribution while $S$ is a third central moment and is a measure of asymmetry about the mean amplitude value of $\mathbf{X}$.

We have set 5-dimensional feature vector with real-valued explanatory variables $\mathbf{V}=\left(P_{H}, P_{L} / P_{H}\right.$, $\left.P_{L} / P_{S}, K, S\right) . \mathbf{V}$ represents points in appropriate multidimensional space and offers characterization

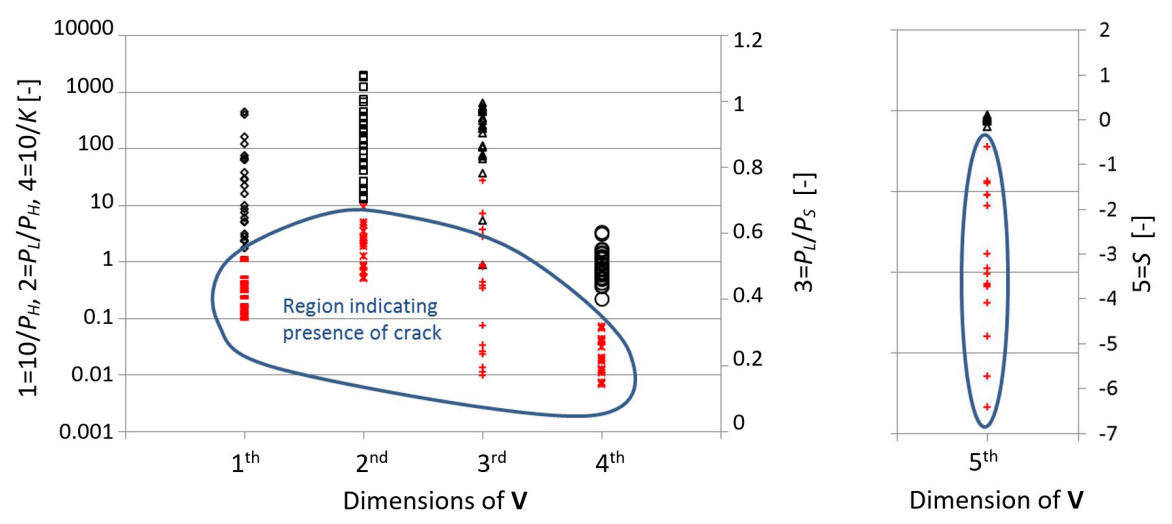

Fig. 5. Damaged mold definition based on feature vector $V$ 
of the process. Fig. 5 show characterization of the injection molding (IM) process regarding the presence of mold defects. Hollow black markers represent vectors $\mathbf{V}$ during production using the new and damaged mold, where the mold pressure is relatively low and is below activation level of defects in the tool steel. Red markers $(-, \times,+$ and $*)$ on Fig. 5 represent the vectors $\mathbf{V}$ during production with the damaged mold, where the mold pressure is above the activation level of defects in the steel. Vectors $\mathbf{V}$ that indicate the presence of damage are positioned in encircled region.

Definition of signal boxes with defined time duration reduces computational complexity during measurement and simplifies the analysis of acquired signals during monitoring. For example in a case of injection molding individual AE signal box can simultaneous carry information closely linked with a process step together with the information of injection mold integrity. AE signal box can thus represent mixture of information that can be with an individual AE signal burst analysis additionally dismembered.

\subsection{Burst Analysis}

Above mentioned disadvantage of the signal boxes analysis that can simultaneous carry information closely linked with a process step together with the information of mold integrity can overcome signal burst analysis. Within the scope of this method we used signal bursts for derivation of signal descriptors that are stacked to form a feature vector $\mathbf{Z}=\left(z_{1}, z_{2}, \ldots\right.$, $\left.z_{73}\right)$. The union of feature vectors is acquired during different phases of process. Together with already described features that represent signal frequency characteristics and signal amplitude probability distribution in feature vector $\mathbf{V}$ in paragraph 3.2 , additional time-domain features are added in a feature vector $\mathbf{Z}$, like $A_{p}$, (peak amplitude), $R_{t}$ (rise time), $d$ (duration) and $R A$ value (ratio of $R_{t} \operatorname{nad} A_{p}$ ). To overcome pitfalls of Fourier analysis, Wavelet analysis is applied for processing signals during cycle. The wavelet packet analysis decomposes signal into several levels in the whole measured frequency band [24] and [25]. To improve frequency resolution the detail of the signal is decomposed as well [26]. The wavelet packet corresponds to some frequency band and includes information on signal in different time windows at different resolutions. For the analysis some packets contain important information while other packets are relatively unimportant.

For the computation of wavelet packet transform of signal $X(t)$ we used algorithm described below:

$$
\begin{gathered}
P_{0}^{1}(t)=X(t), \\
P_{j}^{2 i-1}(t)=H P_{j-1}^{i}(t), \\
P_{j}^{2 i}(t)=G P_{j-1}^{i}(t) .
\end{gathered}
$$

In above equations $P_{j}^{i}(t)$ represents $i^{\text {th }}$ packet on $j^{\text {th }}$ resolution, with time parameter $t=1,2, \ldots, 2^{J-j}$, $i=1,2, \ldots, 2^{j}, j=1,2, \ldots, J, J=\log _{2} N$. Operators $H$ and $G$ are convolution sum defined as:

$$
\begin{aligned}
& H\{\cdot\}=\sum_{k} h(k-2 t), \\
& G\{\cdot\}=\sum_{k} g(k-2 t) .
\end{aligned}
$$

In above equations $h(t)$ and $g(t)$ are a pair of quadrature mirror filters. A time parameter $t$ is taken as a series of integers $k(t \rightarrow k=1,2, \ldots)$.

To define the energy of wavelet packet we used:

$$
E_{j}^{i}=\sum_{i=1}^{2^{J-j}}\left(P_{j}^{i}(t)\right)^{2} .
$$

The energy of wavelet packet on selected resolution $\mathrm{j}$ is normalized as:

$$
R_{j}^{i}=E_{j}^{i} / \sum_{i=1}^{2^{j}} E_{j}^{i}
$$

For the description of AE bursts we have set the feature vector $\mathbf{Z}=\left(P_{H}, P_{L} / P_{H}, P_{L} / P_{S}, K, S, A_{p}, R_{t}, R A, d\right.$, $\left.R_{5}^{1}, R_{5}^{2}, \ldots, R_{5}^{32}, E_{5}^{1}, E_{5}^{2}, \ldots, E_{5}^{32}\right)$.

But the dimension of feature vector should not be arbitrary large. Large vectors increase computational complexity, and causes a decrease of performance [27]. A small set of features can also give us satisfactory classification performance. Feature selection method offers selection of subset $F_{j}(B)=\left\{z_{b} \mid b=1, \ldots, B\right\}$ which surpass other subsets with dimension $B$ as:

$$
J\left(F_{i}(B)\right) \geq J\left(F_{j}(B)\right) \text { for all } j \in\{1, \ldots, q(B)\},
$$

where $q(B)$ is the number of evaluations of performance measure $J\left(F_{j}(B)\right)$. For reduction of dimension of feature vector we have applied sequential forward selection (SFS) method with $k$-Nearest Neighbor ( $k$-NN) classification algorithm. With $k$-NN the input consists of the $k=3$ closest training examples in the feature space.

SFS method offered us selection of feature subset $\mathbf{Z}_{\mathbf{s}}$ with a size of $10 . \mathbf{Z}_{\mathbf{s}}$ is an input for neural network pattern recognition of acquired bursts during the IM production cycle. The most informative selected features based on SFS are stacked in feature subset as follows: $\mathbf{Z}_{\mathrm{s}}=\left(R_{5}^{8}, R_{5}^{16}, R_{5}^{24}, R_{5}^{21}, R_{5}^{18}, R_{5}^{20}, d, R_{5}^{6}, R_{5}^{32}, A_{p}\right)$. Vectors of the training data have been designated into 
categories based on the time moment of their acquisition during the injection molding cycle and unsupervised fuzzy $\mathrm{C}$ means clustering (FCM) for vectors of the damaged mold. FCM clustering was found to be an effective algorithm for clustering of acoustic emission vectors composed of multiple features [28] and [29].

We introduced principal component analysis (PCA) for presentation of feature vectors $\mathbf{Z}_{\mathbf{s}}$. Principal component analysis, as an unsupervised feature reduction method, is mathematically defined as an orthogonal linear transformation that transforms the data into a new coordinate system. Fig. 6 shows the distribution of feature vectors $\mathbf{Z}_{\mathrm{s}}$ where these vectors are divided into five classes. Class 1 represents vectors characteristic for the closing of the mold, Class 2 vectors characteristic for the injection of the melt with maximum pressure, Class 3 vectors characteristic for the holding (packing) stage, Class 4 represents opening of the tool and Class 5 vectors characterizing damage of the mold.

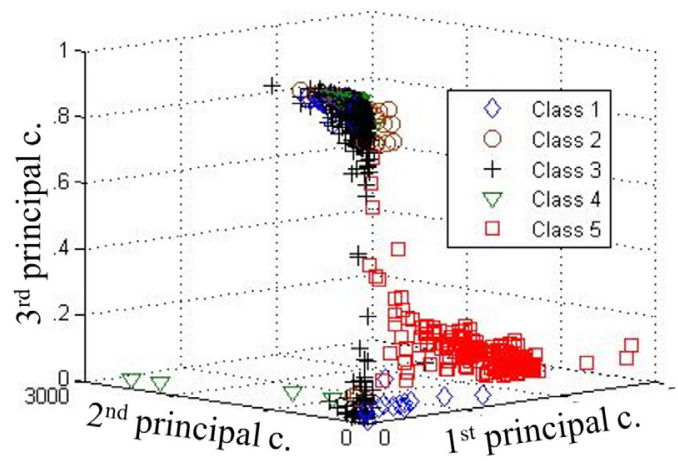

Fig. 6. The distribution of feature vectors $\mathbf{Z}_{\mathbf{s}}$

For automatic classification of vectors $\mathbf{Z}_{\mathrm{s}}$ we introduced neural network pattern recognition. The network was set as feed-forward with 10 neurons in the hidden layer. The transfer function was $\mathrm{F}$ Tan Sigmoid function for the hidden and the output layer. Five output neurons are used, because of five target classes. Scaled conjugate gradient back-propagation was used for training the network.

Neural network pattern recognition offered us on average $91.6 \%$ correctly classified vectors $\mathbf{Z}_{\mathbf{s}}$. The lowest error $E=0.8 \%$, or best classification was for vectors of the Class 3 . These represent processorientated bursts during the holding (packing) phase. $96.7 \%$ correct was detection of damage (vectors of Class 5). The algorithm is not recommended for classification of mold closing with $81 \%$ error, i.e. Class 1 vectors, and classification of mold opening vectors (Class 4 ) with $32 \%$ error.

\section{CONCLUSIONS}

Results of AE monitoring during injection molding of polypropylene specimens are presented. Useful information about the presence of mold cracks in damaged mold area can be obtained by measuring time domain $\mathrm{AE}$ signal parameters during individual injection molding cycle phases. AE signal processing for evaluation of the defects in the injection mold can be achieved also with fractal algorithm using box counting method from time discrete signals. To improve the tool integrity prediction with smaller defects implementation of AE signal analysis based on idea of box counting method is used. Definition of feature vector of $\mathrm{AE}$ signal boxes with defined time duration reduces computational complexity during measurement, for example in production environment and simplifies the analysis of acquired AE signals during monitoring.

But AE signal box can represent mixture of information that can be with an individual AE signal burst analysis additionally dismembered. For this we implemented 10-dimensional feature subset vector with instances that are real-valued descriptors (variables). With neural network pattern recognition this vector offers us defining points in appropriate multidimensional space to characterize the damage in a mold with a high accuracy, and also recognition of acquired $\mathrm{AE}$ brusts that characterize the injection of the melt and holding phase injection molding production cycle.

\section{ACKNOWLEDGMENTS}

The research work was partly financed by the Slovenian Ministry for Higher Education, Science and Technology, European Social Fund (ESF) and by the European Union (EU).

\section{REFERENCES}

[1] Pollock, A.A. (2009). Fundamentals of Acoustic Emission Testing. Moore, P.O., Miller, R.K., Hill, E.V.K. (eds.), Nondestructive Testing Handbook-Acoustic Emission Testing, $3^{\text {rd }}$ ed. American Society for Nondestructive Testing. Columbus, p. 31-108.

[2] Davoodi, S., Mostafapour, A. (2013). Modeling acoustic emission signals caused by leakage in pressurized gas pipe. Journal of Nondestructive Evaluation, vol. 32, no. 1, p. 67-80, Dol:10.1007/s10921-012-0160-x.

[3] Harri, K., Guillaume, P., Vanlanduit, S. (2008). On-line damage detection on a wing panel using transmission of multisine ultrasonic waves. NDT \& E International, vol. 41, no. 4, p. 312317, DOI:10.1016/j.ndteint.2007.10.012. 
[4] Mazal, P., Pazdera, L., Kolar, L. (2006). Basic acoustic emission signal treatment in the area of mechanical cyclic loading. International Journal of Microstructure and Materials Properties, vol. 1, no. 3-4, p. 341-352, D0l:10.1504/ IJMMP.2006.011649.

[5] Cao, J., Luo, H., Han, Z. (2012). Acoustic emission source mechanism analysis and crack length prediction during fatigue crack propagation in $16 \mathrm{Mn}$ steel and welds. Procedia Engineering, vol. 27, p. 1524-1537, D0l:10.1016/j. proeng.2011.12.617.

[6] Mukhopadhyay, C.K., Sasikala, G., Jayakumar, T., Raj, B. (2012). Acoustic emission during fracture toughness tests of SA333 Gr.6 steel. Engineering Fracture Mechanics, vol. 96, p. 294-306, D0I:10.1016/j.engfracmech.2012.08.004.

[7] Drummond, G., Watson, J.F., Acarnley, P.P. (2007). Acoustic emission from wire ropes during proof load and fatigue testing. NDT \& E International, vol. 40, no. 1, p. 94-101, DOI:10.1016/j.ndteint.2006.07.005.

[8] Kim, K.-B., Yoon, D.-J., Jeong, J.-C., Lee, S.-S. (2004). Determining the stress intensity factor of a material with an artificial neural network from acoustic emission measurements. NDT \& E International, vol. 37 , no. 6, p. 423429, D0I:10.1016/j.ndteint.2003.08.007.

[9] Baensch, F. (2014). Damage Evolution in Wood and Layered Wood Composites Monitored in Situ by Acoustic Emission, Digital Image Correlation and Synchrotron Based Tomographic Microscopy. PhD Thesis, ETH Zürich, Zürich.

[10] Podrug, S., Glodež, S., Jelaska, D. (2011). Numerical Modelling of Crack Growth in a Gear Tooth Root. Strojniški vestnik Journal of Mechanical Engineering, vol. 57, no. 7-8, p. 579586, D0I:10.5545/sv-jme.2009.127.

[11] Akrache, R., Lu, J. (2011). Integrated Design for Fatigue Life Estimation of Structures. Strojniški vestnik - Journal of Mechanical Engineering, vol. 57, no. 7-8, p. 547-554, DOl:10.5545/sv-jme.2008.043.

[12] Carboni, M., Bruni, S., Crivelli, D., Guagliano, M., Rolek, P. (2014). A study on the performance of acoustic emission and low frequency vibration methods to the real-time condition monitoring of railway axles. 12th International Conference of the Slovenian Society for The Non-Destructive Testing in Engineering, Journal of Acoustic Emission, vol. 32, p. S1-S20.

[13] Zaki, A., Chai, H.K., Aggelis, D.G., Alver, N. (2015). NonDestructive evaluation for corrosion monitoring in concrete: $\mathrm{A}$ review and capability of acoustic emission technique. Sensors, vol. 15, no. 8, p. 19069-19101, D0l:10.3390/s150819069.

[14] Momon, S., Godin, N., Reynaud, P., R'Mili, M., Fantozzi, G. (2012). Unsupervised and supervised classification of $\mathrm{AE}$ data collected during fatigue test on $\mathrm{CMC}$ at high temperature. Composites Part A: Applied Science and Manufacturing, vol. 43, no. 2, p. 254-260, D0l:10.1016/j. compositesa.2011.10.016.

[15] Aggelis, D.G. (2011). Classification of cracking mode in concrete by acoustic emission parameters. Mechanics Research Communications, vol. 38, no. 3, p. 153-157, D0I:10.1016/j.mechrescom.2011.03.007.

[16] Svečko, R., Kusić, D., Kek, T., Sarjaš, A., Hančič, A., Grum, J. (2013). Acoustic emission detection of macro-cracks on engraving tool steel inserts during the injection molding cycle using PZT sensors. Sensors, vol. 13, no. 5, p. 6365-6379, DOl:10.3390/s130506365.

[17] Marec, A., Thomas, J.-H., El Guerjouma, R. (2008). Damage characterization of polymer-based composite materials: Multivariable analysis and wavelet transform for clustering acoustic emission data. Mechanical Systems and Signal Processing, vol. 22, no. 6, p. 1441-1464, Dol:10.1016/j. ymssp.2007.11.029.

[18] Požar, T., Možina, J. (2014). Detection of subnanometer ultrasonic displacements. Sattler, K.D. (ed.), Fundamentals of Picoscience; CRC Press, Boca Raton, p. 553-578.

[19] ISO 294-3 (2002). Plastics -- Injection Moulding of Test Specimens of Thermoplastic Materials -- Part 3: Small Plates. International Organization for Standardization, Geneva.

[20] Kumar, J., Punnose, S., Mukhopadhyay, C.K., Jayakumar, T., Kumar, V. (2012). Acoustic emission during tensile deformation of smooth and notched specimens of near alpha titanium alloy. Research in Nondestructive Evaluation, vol. 23, p. 17-31, DOI:10.1080/09349847.2011.622068.

[21] Hase, A., Mishina, M., Wada, M. (2012). Correlation between features of acoustic emission signals and mechanical wear mechanisms. Wear, vol. 292-293, p. 144-150, D0l:10.1016/j. wear.2012.05.019.

[22] Shenoi, B.A. (2006). Introduction to Digital Signal Processing and Filter Design. John Wiley \& Sons, Hoboken, New Jersey.

[23] Župerl, U., Čuš, F., Irgolič, T. (2016). Prediction of Cutting Forces in Ball-End Milling of Multi-Layered Metal Materials. Strojniški vestnik - Journal of Mechanical Engineering, vol. 62, no. 6, p. 340-350, Dol:10.5545/sv-jme.2015.3289.

[24] Aijun Yin, Juncheng Lu, Zongxian Dai, Jiang Li, Qi Ouyang, (2016). Isomap and Deep Belief Network-Based Machine Health Combined Assessment Model. Strojniški vestnik Journal of Mechanical Engineering, vol. 62, no. 12, p. 740750, DOl:10.5545/sv-jme.2016.3694.

[25] Zhu, K., , Wong, Y.S., Hong, G.S. (2009). Wavelet analysis of sensor signals for tool condition monitoring: A review and some new results. International Journal of Machine Tools and Manufacture, vol. 49, no. 7-8, p. 537-553, D0l:10.1016/j. ijmachtools.2009.02.003.

[26] Wang, X.H., Zhu, C.M., Mao, H.L., Huang, Z.F. (2009). Wavelet packet analysis for the propagation of acoustic emission signals across turbine runners. NDT \& E Internnational, vol. 42, no. 1, p. 42-46, Dol:10.1016/j.ndteint.2008.07.005.

[27] Van der Heijden, F., Duin, R.P.W., de Ridder, D., Tax, D.M.J. (2002). Classification, Parameter Estimation and State Estimation, John Wiley \& Sons, West Sussex.

[28] Oskouei, A.R., Heidary, H., Ahmadi, M., Farajpur, M. (2012). Unsupervised acoustic emission data clustering for the analysis of damage mechanisms in glass/polyester composites. Materials \& Design, vol. 37, p. 416-422, D0I:10.1016/j.matdes.2012.01.018.

[29] Kek, T., Kusić, D., Grum, J. (2016). Wavelet packet decomposition to characterize injection molding tool damage. Applied Sciences, vol. 6, no. 2, p. 45-57, D0l:10.3390/ app6020045. 